\title{
NOTES D'ACTUALITÉ
}

\section{Soutenir l'innovation sociale pour construire une société soucieuse du bien commun}

\author{
Le Réseau québécois en innovation sociale (RQIS) ${ }^{\mathrm{a}}$
}

Au-delà de la crise sanitaire qu'il provoque actuellement dans l'ensemble de la planète, la COVID-19 est le révélateur d'une crise plus profonde qui corrode nos sociétés. Cette crise est due à l'affaiblissement progressif des liens de solidarité et de réciprocité causé par le modèle économique qui nous gère. Il suffit de voir le sort que nous accordons aux personnes âgées et le traitement qu'on leur dispense dans les CHSLD pour s'en rendre compte. Nul doute que les voies de sortie de la pandémie qui affecte tous les aspects de nos vies passeront par des recherches dans le domaine de la santé, mais aussi des communications, de la gestion, etc. Dans tous ces domaines, des innovations seront nécessaires, qui mobiliseront à leur tour des recherches et des initiatives dans les domaines technologique et sanitaire, mais une riposte plus définitive, plus globale, doit se diriger à la source du problème, soit le modèle social et économique actuel. En cette matière, une réflexion approfondie sur notre avenir et sur les innovations qui le jalonnent s'avère nécessaire afin de construire un avenir plus prometteur pour tous.

Au cours des dernières années, stimulées par la recherche de la croissance économique et de la rentabilité financière, les innovations technologiques ont connu un essor considérable. En témoigne le développement des outils de communication, de l'industrie 4.0 et de l'intelligence artificielle dont les impacts se font sentir dans les différentes facettes de nos vies : transport, relations humaines, enseignement, etc. Partout, de nouveaux dispositifs se succèdent afin de faciliter - supposément - nos existences. En même temps, l'humanité s'engouffre dans une catastrophe écologique sans précédent. Comme pour la pandémie actuelle, la technologie devra être mobilisée pour y répondre, mais la vraie réponse sera avant tout sociale. Pour accroitre le bien-être collectif, l'innovation technologique doit prendre en compte l'impact qu'ont les nouveaux usages sur les personnes, les organisations, les systèmes en place. Elle doit se combiner à des expérimentations sociales puisque c'est notre façon de vivre ensemble, d'utiliser et de partager les ressources existantes qu'il nous faut revoir.

Force est de constater que, au cours des dernières années, la dimension sociale a été le parent pauvre des efforts financiers de nos gouvernements tant municipal, provincial que fédéral pour soutenir l'innovation. En comparaison des sommes dévolues à l'innovation technologique, celles consacrées à l'innovation sociale ont été plus que modestes. Dans les politiques publiques, l'innovation sociale est souvent perçue comme étrangère et sans liens avec l'innovation technologique. N'est-il pas le temps de changer cette vision et de réaliser que cette séparation du technologique et du social n’a plus de sens?

Le Réseau québécois en innovation sociale (RQIS) se fait depuis plus de quinze ans le porteur d'une perspective large de l'innovation, une perspective qui se nourrit des nombreuses innovations sociales qui prennent forme sur le territoire québécois, tant comme réponses à des enjeux sociétaux que comme lieux de création et de collaboration. Porter attention à l'innovation sociale, c'est réaliser que la technologie n'est pas une panacée et qu'elle doit être accompagnée d'une réflexion sur ces impacts sociaux et organisationnels.

${ }^{\mathrm{a}}$ Voir notes de fin pour plus de détails ainsi que pour la liste des signataires 
Porter attention à l'innovation sociale, c'est aussi la considérer comme une composante essentielle d'un écosystème d'innovation et d'une société soucieuse du bien commun.

En ce début de sortie de crise dont les résultats sont encore incertains, le RQIS et ses membres invitent les diverses instances gouvernementales à franchir un pas vers une société de plus en plus soucieuse du bienêtre collectif, assumant son interdépendance et plus résiliente parce que plus inclusive et solidaire.

Nous recommandons ainsi aux pouvoirs publics

- de soutenir davantage les initiatives mettant en valeur des innovations sociales dans toutes les sphères d'activité au cours de la prochaine phase de relance;

- de participer activement aux discussions à venir organisées par de nombreux réseaux sur les façons de soutenir l'innovation sociale y compris celles faisant usage de technologies, dans les prochaines années et de s'inspirer de ces discussions pour améliorer les politiques publiques;

- d'intégrer dans l'ensemble des programmes gouvernementaux un soutien spécifique à l'innovation sociale (en santé, en éducation, en économie, en développement territorial, etc.)

\title{
NOTE
}

Le Réseau québécois en innovation sociale (RQIS) est un espace de partage et de mobilisation des savoirs et des expériences qui contribue à faire de l'innovation sociale un levier indispensable au développement du Québec. Ses membres proviennent des milieux de la recherche, de l'intervention, du développement et du transfert de connaissances et ils œuvrent dans les secteurs économique, social, de l'éducation et de la culture.

\author{
Liste des signataires \\ Arbour, Nadine ÉCOBES Collège de Jonquière \\ Beaudoin, Josée L'École en réseau \\ Bouchard, Marie J. UQAM, Département d'organisation et ressources humaines \\ Brown, Hélène Percolab \\ Dubé, Patrick Maison de l'innovation sociale \\ Dubé, Stéphane Institut du Nouveau Monde \\ Dancause, Luc Sapiens conseils \\ Ducharme, Roch Centre de recherche pour l'inclusion des personnes en situation de handicap (CRISPESH) \\ Élissade, Jérôme Perspectiv \\ Fortin, André Créativité 33 \\ Frappier, Martin Chantier de l'économie sociale \\ Grenier, Pascal, $\quad$ Esplanade \\ Gulian, Thomas Institut de recherche sur l'intégration professionnelle des immigrants (IRIPI) - Collège de Maisonneuve \\ Klein, Juan-Luis UQAM, Département de géographie \\ Lagacé, Marie Claude Humanov-is \\ Légaré, Catherine Academos \\ Lemire, Nicole Travailleuse autonome \\ Lévesque, Benoît UQAM, Département de sociologie \\ Mahy, Isabelle UQAM, Département de communication sociale et publique \\ Mailhot, Chantale HEC Montréal, Département de management
}




$\begin{array}{ll}\text { Michaud, Dominique } & \text { Université Concordia } \\ \text { Morisette, Martine } & \text { Carrefour Jeunesse emploi de l'Outaouais } \\ \text { Robitaille, Martine } & \text { Université du Québec en Outaouais (UQO) } \\ \text { Simoneau, Marcel } & \text { UQAM, Service aux collectivités } \\ \text { St-Pierre, Linda } & \text { Centre de transfert sur la réussite éducative du Québec (CTREQ) } \\ \text { Tousignant, Élise } & \text { Centre d'étude en responsabilité sociale et écocitoyenneté (CERSÉ) - Collège de Rosemont } \\ \text { van Schendel, Vincent } & \text { Territoires innovants en économie sociale et solidaire (TIESS) }\end{array}$

